\title{
Räumliche Transformationen: Prozess, Ziel, Leitbild?
}

Markus Hesse

Ziel dieses Beitrags ist es, »Transformation « in die raum- und planungsbezogene Praxis einzuordnen, hier vor allem in die Debatte um raumbezogene Leitbilder. Der Fokus auf Leitbilder erfolgt hier deshalb, weil die Rede von der Transformation mittlerweile zumindest im deutschsprachigen Raum eine hohe Breitenwirkung erzielt hat. Der Begriff »Transformation« hat mindestens implizit, wenn nicht sogar explizit, Leitbildcharakter (WBGU 2011). Entsprechendes gilt, wenn auch inhaltlich anders akzentuiert, im internationalen Sprachraum für Sustainability Transitions (Frantzeskaki/Broto/Coenen et al. 2017). Beide Diskurse sind zunehmend relevant für eine mögliche Operationalisierung von Pfaden einer Postwachstumsentwicklung.

Die erkenntnisleitende Frage dieses Beitrags ist: Kann raumbezogene Transformation als Leitbild betrachtet werden, und wenn ja, worin äußert sich dies konkret in analytischer, normativer oder prozeduraler Hinsicht? Hat Transformation einen allgemeinen Deutungsanspruch oder ist sie spezifisch ausgerichtet - was ist die konkrete Zielformulierung für welche Ebene? Oder ist Transformation primär als prozedurale Richtschnur zu verstehen, als Metapher zur kollektiven Mobilisierung von Wandel, deren Inhalte hinter den zuweilen recht aufwendigen partizipativen Prozessen eher verborgen bleiben? Bevor zu diesen Fragen einige Antwortversuche gemacht werden, sollen die beiden im Zentrum stehenden Begriffe kurz diskutiert werden.

\section{(Große) Transformation}

Der erste Fixpunkt hier sind Große Transformation (GT) bzw. deren semantische Schwester Sustainability Transitions (STs), die seit Ende der 2010er Jahre in Forschung und Praxis überaus populär geworden sind. Mit Blick auf die 
Rezeption der ideengeschichtlichen Quelle des »Großen« im Transformationsdiskurs geht es hier natürlich um das Werk von Karl Polanyi (1944). Das Buch enthält eine überaus anregende Zusammenschau von gesellschaftlicher, wirtschaftlicher und politischer Entwicklung. Im Kern geht es dabei um zwei Dinge: zum einen um die Erfahrung des Niedergangs liberaler politischer Verfassung im Zeichen autoritärer Regime, zum anderen um die unter dem Begriff der »Doppelbewegung « eingebrachte Spannung zwischen Marktliberalismus und Demokratie. Polanyi hat diese Spannung als prinzipiell nicht auflösbar angesehen; denn ein großmaßstäblicher politischer Umbau lässt sich womöglich nur begrenzt mit demokratischen Prinzipien und Praktiken in Einklang bringen. Abschnitt 3 seines Buchs befasst sich konsequenterweise mit den Bedingungen von Freiheit in komplexen Gesellschaften. Gleichwohl ist die Überlegung elementar für Polanyis Denkansatz, dass wirtschaftliche Dynamik durch einen aktiven, interventionistischen Staat gesellschaftlich »eingehegt« oder rückgebettet werden muss.

(Große) Transformation bezieht sich in dieser Tradition heute ebenfalls auf die Rückbettung entfesselter technologischer und wirtschaftlicher Dynamiken in die Gesellschaft. Gemeint ist damit der »massive ökologische, technologische, ökonomische, institutionelle und kulturelle Umbruchprozess (Schneidewind 2018), vor dem die Welt zu Beginn des 21. Jahrhunderts steht. Nur durch diesen Umbruch ließen sich die vielen Krisen des industriellen Natur-, Wirtschafts- und Gesellschaftssystems lösen. Insofern repräsentieren Transformation und Sustainability Transitions mittlerweile eine Art Mainstream der aktuellen Umwelt-, Technologie- und Nachhaltigkeitsforschung (Zolfagharian/Walrave/Raven et al. 2019). Wie die Themenwahl des ARL-Kongresses 2019 demonstriert hat, der diesen Sammelband veranlasst hat, ist Transformation mittlerweile auch im stadt- und raumbezogenen Diskurs etabliert. Besonders inspirierend speziell an der Transitions-Debatte ist sicher die geschärfte Sensibilität für evolutionären Wandel, denn in ihrem Zentrum steht die Suche nach Übergängen (!) von real existierenden Situationen zu favorisierten bzw. als notwendig erachteten Zuständen. Damit verbindet sich die Frage an alternative Diskurse, wie aus Nischen- und Pionierkonzepten verallgemeinerbare Strategien in Richtung Nachhaltigkeit erwachsen können.

Eine enorme selbstverstärkende Wirkung für die Popularisierung der GT hatte die Berichterstattung des Wissenschaftlichen Beirats zu Globalen Umweltveränderungen der deutschen Bundesregierung (WBGU 2011, 2016). 
Der WBGU hat Große Transformation bereits im 2011er-Hauptgutachten "Welt im Wandel« behandelt und sehr emphatisch zum Thema eines »Gesellschaftsvertrags « gemacht. In seinem 2016er-Gutachten »Der Umzug der Menschheit: Die transformative Kraft der Städte« wurde Transformation zum ersten Mal in einer spezifischen raum- und stadtbezogenen Perspektive gedacht. Das Gutachten legt den Fokus auf Urbanisierungsprozesse und Städte. Es hat damit globale sozialökologische Zusammenhänge mit der Frage von Urbanisierungsprozessen verknüpft. Allerdings ist die Argumentation des WBGU nicht frei von kausalen Fehlschlüssen. Dies gilt vor allem für die spezifische Konstruktion des Städtischen: Die Tatsache, dass eine Mehrheit der Bevölkerung in Städten lokalisiert ist, heißt noch lange nicht, dass das Problem im Kern in den Städten bzw. durch die Städte gelöst werden könnte respektive primär anvisiert werden müsste. Diesen Blickwinkel haben Angelo und Wachsmuth (2015) auch als smethodological cityism< kritisiert; der Fokus auf die Städte verkennt die überragende Rolle, die Nationalstaaten und supranationaler Regulierung auch im sogenannten Zeitalter der Städte zukommt.

Um die annoncierten Ziele der Großen Transformation im urbanen Kontext zu erreichen, formuliert das Gutachten des WBGU (2016) anspruchsvolle normative Setzungen. Bei der Umsetzung transformativer Strategien haben städtische Räume - und darin die räumliche Planung - praktisch eine Schlüsselrolle. "Wir brauchen die Raumplanung!«, war das entsprechende Credo von Dirk Messner, als er das Gutachten in einer Keynote auf der Dortmunder Konferenz für Raum- und Planungsforschung 2018 vorstellte. Das mehrheitlich aus Vertreter*innen der raumbezogenen Planung und Forschung bestehende Publikum wollte diesem Diktum indes nicht ohne Weiteres folgen - eine Skepsis, die sich auf die realistische Beurteilung von Stellenwert und faktischem Leistungsvermögen der räumlichen Planung stützen dürfte. In politik- und planungstheoretischer Hinsicht gewissermaßen delikat ist, dass der WBGU inkrementelle Lösungen verwirft, zugunsten des ganz großen Wurfs - ohne dass man aber den bzw. die jeweiligen Träger, Strategien und Instrumente hierfür ausfindig machen könnte: »Innerhalb weniger Jahre muss in den Städten ein Paradigmenwechsel stattfinden: weg von inkrementellen Ansätzen, hin zu transformativen Änderungen, um langfristig die natürlichen Lebensgrundlagen der Menschheit und die Lebensqualität der Menschen zu erhalten.«(WBGU 2016, S. 20) Andere Autor*innen sehen GT dagegen durchaus in der Tradition eines inkremen- 
tellen Planungsverständnisses, innerhalb dessen mit adaptiven Strategien auf den zunehmend disruptiv erfolgenden Wandel reagiert wird (Iwaniec/ Cook/Barbosa et al. 2019).

Mit der Terminologie des Großen hat sich das WBGU-Gutachten explizit auf die politisch-ökonomische Denktradition Polanyis bezogen. Umso verwunderlicher ist, dass der Ansatz ausgerechnet auf diesem Terrain seine größten Schwächen hat: »Von einer großen Transformation im Sinne Polanyis könnte nur die Rede sein, wenn nach Alternativen zu dem selbstregulierenden Marktsystem und zu marktkonformen Anpassungsmaßnahmen gefragt wird. Legen wir diesen Maßstab zugrunde, so wird die Halbherzigkeit des Polanyi-Bezugs des WBGU wie auch vieler anderer Beiträge zur Transformationsforschung deutlich." (Thomasberger 2016: 34) Auch der Urbanisierungsbericht des WBGU von 2016 bleibt vage dahingehend, wer eine so umfassende Aufgabe wie gesellschaftliche Transformation in relativ kurzer Zeit stemmen kann - die Weltgesellschaft, Pioniere des Wandels, Schlüsselakteure? Er enthält wenig Kritisches zu Institutionen. Allgemeine Aussagen zu Grund und Boden entbehren robuster Vorschläge dahingehend, wie eine Neuorientierung von Eigentumsverhältnissen erstens implementiert werden könnte und wie zweitens die damit zwangsläufig verbundenen Friktionen und Konflikte gelöst werden könnte. Schneidewind (2018) ist diesbezüglich konkreter und reflektiert auch die Rahmenbedingungen der politischen Ökonomie. Seine Überlegungen sind indes nicht zwingend leichter umsetzbar: Dort sind es im Grunde alle Ebenen und Akteure der Transformation, die nur richtig miteinander verzahnt werden müssen.

Das Redesign lokaler Praktiken als genuin transformatives Handeln bringt zweifellos eine Blutauffrischung für die Politik, die sich in ihren administrativen Routinen, verfestigten Interessenkonflikten und speacemeak-Lösungen erkennbar in Sackgassensituationen manövriert hat. Die Versuchung, derartige Dilemmata mit einem großen Wurf zu überwinden, ist naheliegend. Praktizierte Transformation muss ihre Wirkung - und ihre immanenten Vorteile gegenüber bisherigen Praktiken - meines Erachtens allerdings erst noch beweisen. Und zurecht gibt es Fragen an die nahezu inflationäre Praxis von Reallaboren: Zumindest ist unklar, wie genau bestehende Institutionen in neue Praktiken und Ansätze einbezogen werden. Und mit Blick auf die ebenso inflationäre Forderung nach Beteiligung der Öffentlichkeit in welcher Transformation auch immer - gegen die prinzipiell keine Einwände zu erheben sind - ließe sich doch die folgende Frage hinzu- 
fügen: Was tut man, um die Probleme der Zufälligkeit, des Erratischen und der Partikularinteressen zu vermeiden, die sich in der Regel im "Albtraum Partizipation« (Miessen 2012) eben auch vorfinden lassen?

\section{Leitbilder}

Der zweite Fixpunkt dieses Beitrags sind Leitbilder - vor dem Hintergrund der Annahme, dass Große Transformation mittlerweile einen solchen Stellenwert hat. Konsens dürfte sein, dass GT weder Ziel an sich ist noch geht es in erster Linie um den Prozess als solchen, auch wenn man gelegentlich diesen Eindruck gewinnen kann. GT bündelt in ihrem umfassenden Ansatz ein Leitbild, eine Art utopisches Narrativ (Giesel 2007; Dahlstrom 2014; Zieschank/Ronzheimer 2017). Sie bringt Alternativen zum Bestehenden in einen auf den ersten Blick konsistenten Rahmen und verknüpft auf der Ebene der Problematisierung durchaus übergreifende Fragestellungen (etwa die Wachstumsfrage) mit praktischen bzw. lokalen Strategien. In raumbezogener Hinsicht baut GT dabei auf den bekannten Elementen nachhaltiger Stadtund Raumentwicklung auf und verknüpft diese mit dem Narrativ eines großen Ganzen. Dem Konstrukt »Transformation" geht es dabei aber wie allen Vorgängerleitbildern: De facto bilden sie eine eher widersprüchliche Mischung aus Lenkungs- und Entwicklungszielen, aus raumbezogenem oder sektoralem Fokus, schließlich dezidiert auch aus prozeduralen Elementen. In der Praxis sind sie eher heterogen, immer hochgradig abstrakt, um auf diese Weise eine gewisse Hegemonie oder Mehrheitsfähigkeit zu erlangen. Und im Zeitablauf haben sie sich mindestens als wechselhaft herausgestellt, gelegentlich auch opportunistisch, getrieben vom Zeitgeist (Hesse/Leick 2013). Man folgt nicht nur spezifischen Problemwahrnehmungen oder dem Wettbewerb um Fördergelder, sondern ist auch abhängig von temporären Moden, Konjunkturen und Konstrukten.

Dies lässt sich leicht im Rückblick auf die raumpolitischen Diskurse der 2000er und frühen zo10er Jahre erkennen, die bereits zwei größere »Turns" in ihrer jeweiligen Ausrichtung erlebt haben, zumindest im deutschsprachigen und europäischen Kontext. Seit Mitte der 1990er Jahre dominierte in vielen europäischen Ländern statt der traditionellen Zielvorgabe »räumlicher Ausgleich « ein Fokus auf Wachstum; dies gilt auch für Länder mit einer dezidiert etatistischen Planungstradition wie Großbritannien, die Niederlande 
oder Deutschland. Der Fokus auf Wachstum wurde dann assoziativ - nicht unbedingt empirisch - mit Großstadt- bzw. Metropolregionen verbunden, und zwar aufgrund deren vermeintlicher Rolle als Motoren der wirtschaftlichen Entwicklung (Aring/Sinz 2006). Aufgrund der de facto vielschichtigen Entwicklungsverläufe von Großstadtregionen (sowohl der metropolitanen Räume als auch mittelgroßer Stadtregionen) und veränderter politischer Wahrnehmungen hat sich der Blick in der laufenden Dekade auf Peripherisierungsprozesse und -räume verschoben, insbesondere auf ländliche Räume, Peripherregionen sowie Standorte mit negativer Bevölkerungs- und Wirtschaftsentwicklung. Nun geht es auch verstärkt um splaces left behind, nicht zuletzt, weil deren Bevölkerung sich verstärkt politisch abweichend artikuliert hat (Rodríguez-Pose 2018). In jüngster Zeit werden Leitbilddiskurse um Heimat und raumbezogene Identität ergänzt, gelegentlich auf gehaltvolle Weise, gelegentlich auch im Rahmen eher platter Attitüden und Assoziationen. In dieser Konstellation erscheint GT als der verbindende Überbau, der ganz große Ansatz im Kontext aktueller Narrative zu "grün« und »smart«.

Was lehren diese Erfahrungen im Leitbild-Framing von politisch-planerischen Strategien für die GT? Wovon haben wir bei den "großen« Herausforderungen auszugehen? Geht es um gewissermaßen säkulare Probleme, auf die man mit dem großen Besteck reagieren muss, mit dem das Leitbild durchkomponiert und entsprechend auch die Praxis munitioniert wird ...? Oder gäbe es im Licht der kritischen Selbstbeschreibung von raumbezogener Planung eher gute Gründe zur Zurückhaltung (vgl. Lamker/Levin-Keitel 2019 sowie weitere Beiträge dort)? Auf den ersten Blick betrachtet spricht viel dafür, große Narrative in die Realwelt zu bringen, auch weil die Problemlage dies offenbar erfordert. Zugleich ist aber Vorsicht geboten: Man muss wissen, vor welchen Herausforderungen man steht und wie man mit Risiken umgehen will (Blythe/Silver/Evans et al. 2018). Entsprechend sollte der Diskurs um GT nicht nur pluralisiert, sondern normativ abgerüstet werden: Man kann die Größe der Herausforderung nämlich sicher angemessen präzise beschreiben, ohne in der Praxis gleich jede Demut vor den Anforderungen an politisch-planerische bzw. gesellschaftliche Gestaltung zu verlieren. Wo normativer Überschuss in positivistische Fallen gerät oder vorschnelle Lösungsversprechen macht, ist das auch nicht gut für die Planung. 


\section{Kritische Bewertung}

Was heißt das für Wissenschaft und Praxis? Der Volkswirt Frank Beckenbach hat den gezielten Wandel von der Transformation der Gesellschaft (oder präziser: von der sich transformierenden Gesellschaft) zur Transformationsgesellschaft auch als »Transformationsillusion« bezeichnet (Beckenbach 2017). Begriff und Konzept würden Erwartungen in Richtung auf eine gesellschaftliche Gestaltung wecken, die ziemlich unrealistisch seien, und zwar bezogen auf gleich drei Aspekte: Beckenbach nannte eine Planungsillusion (Prognostizierbarkeit und Steuerbarkeit komplexer Gesellschaften), eine Regulierungsillusion mit Blick auf das gerichtete Zusammenwirken von Marktakteuren, Staatsakteuren und zivilgesellschaftlichen Akteuren und schließlich eine Akzeptanzillusion hinsichtlich der Bereitschaft von mehr als Nischen der Gesellschaft, sich auf einen solchen Pfad einzulassen. Sein Resümee, das er mit Blick auf die Wissenschaft (Ökonomik) konkretisierte: »Große Transformation< ist ungeeignet als Leitlinie.« Der Soziologe Armin Nassehi (2019) hat dies jüngst in einem lesenswerten Interview mit der taz stärker alltagssprachlich ausgedrückt: »Ziele formulieren kann jeder«, heißt es dort etwas süffisant zur einen Seite der Medaille. Robuste Strategien zu formulieren, diese in komplexen Gesellschaften zu implementieren und ihre Wirkung einigermaßen zuverlässig abzuschätzen, sei dagegen sehr viel anspruchsvoller. Hier zeigt sich wohl die eigentliche Herausforderung gezielter Transformationspolitiken.

Dies leitet über zur Beobachtung, dass Transformationsdebatten im Kern immer noch stark von den Logiken und Anforderungen der Forschung durchdrungen erscheinen, was man auch an der schieren Menge einschlägiger Publikationen ablesen kann. Im Vergleich dazu sind neue Pfade in Richtung veränderter Praktiken noch stark unterentwickelt. Oder wie es Koch, Kabisch und Krellenberg (2018: 13) in ihrem Review ausgedrückt haben:

»While the normative understanding of urban transformations has gained considerably importance in urban-related studies and even first steps towards a transformative turn can be identified, this is not reflected in current development processes in cities [...]. An implementation gap between the theoretical concept and the empirical cases is clearly visible.« 
Man findet in der Literatur zudem hinreichend viele Belege für Implementationsprobleme, mit denen sich normative Konzepte wie Nachhaltige Entwicklung schon lange konfrontiert sehen. Zudem sind viele Transformationspfade, die auf sektoraler Ebene konkretisiert werden, so neu nicht, als dass sie die Diskussion zwingend bereichern würden oder eine effektivere Wirkung versprechen würden. Typisch scheint, dass sich entsprechende Auflistungen nicht aktiv mit Erfahrungen aus der Vergangenheit auseinandersetzen, die Implementationsbarrieren gezielten Wandels nicht thematisieren.

Insofern ließe sich fragen, was eigentlich das genuin Neue an Transformation ist -, sieht man von den stark gestiegenen Ambitionen in Richtung gesellschaftliche Steuerung ab? Neu ist meines Erachtens erstens das spezifische Verhältnis von Forschung und Praxis. Die Wissenschaft nimmt hier die Rolle eines engagierten Akteurs ein, der sich auch narrativ positioniert und sich als problemorientiert statt verstaubt sowie transdisziplinär statt traditionell präsentiert. Natürlich sind Wissensproduktion und -dissemination elementar für jeden Wandel. Und doch wirft diese Entwicklung auch Fragen auf. Mit dem missionarischen, geradezu religiösen Ansatz mancher Apologeten der totalen Transformation begibt sich Wissenschaft meines Erachtens auf dünnes Eis - sie macht sich abhängig von guten Absichten und Interessen. Dies muss bekanntlich nicht immer gut enden. Wissenschaftstheoretische Argumente sprechen hier meines Erachtens für mehr Skepsis, vielleicht auch Zurückhaltung. Ich teile auch ausdrücklich nicht die Einschätzung, dass Hochschulen ihre dritte Mission - also neben Forschung und Lehre auch "Transfer" zu befördern - gleich zur ersten Mission machen sollten und jede Praxis diesem Ziel unterordnen (Schneidewind 2018, Abschnitt 21). Eine solche Instrumentalisierung der Forschung dürfte nicht nur Transformation kaum realistischer machen, sondern auch der Wissenschaft schaden. In seiner Stellungnahme zum Umgang mit »Großen gesellschaftlichen Herausforderungen« hat der Wissenschaftsrat (2015) dieses Problem ausführlich thematisiert. Hohe Komplexität, globale Dimensionierung und disziplinäre Komposition der neuartigen Probleme machen die Frage nach der richtigen wissenschaftspolitischen und -strategischen Positionierung kompliziert. Dies hat auch wiederholt Anlass zur kritischen Auseinandersetzung im sinner circle der Transformationsforschung gegeben (vgl. Grunwald 2015, Strunz/Gawel 2017; Grunwald 2018). Vielleicht ist es das Zusammenspiel von zwei Idealtypen von Akteuren, das eine veränderte Praxis ermög- 
lichen könnte: die Positionierung der Forschung als `honest broker (Grunwald 2018) im Sinne von Roger A. Pielke (2007) sowie das Verständnis von Praxis als Handeln reflexiver Praktiker*innen (Schön 1983). Möglicherweise liegen in dieser Kombination tragfähige Ansätze. Allerdings erfordert eine solche Positionierung auf beiden Seiten »ein hohes Maß an Reflexivität und argumentativer Transparenz, Lernfähigkeit nicht nur in analytisch-empirischer, sondern auch normativer Hinsicht und das ständige Infragestellen bisheriger Positionen« (Grunwald 2018: 116).

Neu wäre zweitens, wenn sich die Transformationsforschung ausführlicher mit den Rahmenbedingungen, Potenzialen und Barrieren der Implementation ihrer Vorschläge auseinandersetzen würde (vgl. Dörre/Rosa/ Becker et al. 2019). Dies ist auch der Nachhaltigkeitsforschung bisher nicht gelungen. Sie teilt mit dem Transformationsansatz das Problem, dass die Ausweitung des normativen Zielhorizonts nicht automatisch zu einer Steigerung ihrer Wirkungsweise geführt hat. Welche Anpassungsleistungen müssten Institutionen erbringen, wie sind soziale Sicherungssysteme auf neue Erfordernisse hin umzubauen, welche Folgen hätte Systemtransformation für die politische Willensbildung, für Verteilungsgerechtigkeit? Welche harten Einschnitte sind zu erwarten, die ggf. mit welchen Gewinnen kompensiert werden könnten? Statt der euphemistischen Rede vom großen Umbruch bedarf es meines Erachtens eines nüchternen Blicks auf politische Realitäten (vgl. Bettini/Arklay/Head 2017). Wertvolle Anregungen könnte auch das etablierte politikwissenschaftliche Feld der Transformationsforschung liefern, das im Zuge der politischen Systembrüche von 1989/90 wieder in den Fokus geraten ist (vgl. Kollmorgen/Merkel/Wagener 2015). Die Transformationsforschung befasst sich traditionell mit der Bedeutung von Systemwandel (politisch, ökonomisch) auf Institutionen, Wirtschaftsweisen, Praktiken der politischen Regulierung oder Lebenswelten. Nimmt man die Überblicksdarstellung bei Kollmorgen, Merkel und Wagener (2015) zum Maßstab, dann gibt es hier zahlreiche Anregungen zur Weiterentwicklung der Diskussion. Umgekehrt weist diese sehr umfassende Darstellung noch wenig Bezüge zur ökologischen Transformation auf, erst recht nicht zur Raumentwicklung. Beide Diskurse hätten es jedoch verdient, sich stärker gegenseitig zu durchdringen. 


\section{Schlussfolgerungen}

Mit Blick auf die Leitbildfrage liegt der Charme von "Transformation« womöglich darin, dass dieser Begriff eine angemessen differenzierte Vorstellung von evolutionären gesellschaftlichen Veränderungen bietet. Das ist ein echter Fortschritt, diese analytische Dimension des Begriffs ist überzeugend und sollte weiter vertieft werden. Weitgehend unklar bleibt dagegen meines Erachtens, ob es zum Konzept reicht, um intendierten Wandel auf robuste Weise anzuleiten - also ob Transformation mehr Substanz zu bieten hat als etwa Nachhaltige Entwicklung. Nichts gegen den experimentellen, offenen und subversiven Charakter von Transformation an sich. Wer sich indes auf die Ebene der Großen Transformation begibt, der muss mehr bieten als nur die Summe vieler Einzelmaßnahmen und Spiegelstriche, wo alles enthalten ist, was man sich denken kann, vielleicht auch hier und da bereits gehört hat, und gelegentlich eine radikale Pose hat. Ich folge hier Ulrich Brands (2016) Diktum, dass der strategische Gebrauch von »Transformation« nicht notwendig dazu beiträgt, die vielfältigen Krisen dieser Zeit zu lösen. Dies gilt erst recht für den inflationären Gebrauch dieses Begriffs - wenn also die Spezifika dieses Ansatzes in einer Melange aus allem und jedem verschwimmen und damit auch die möglichen Potenziale einer nicht nur begrifflichen, sondern auch inhaltlichen Neubestimmung gesellschaftlicher Veränderungsdynamiken und entsprechender Politiken eher im Unklaren bleiben.

Will man glaubwürdig, nicht nur metaphorisch, die große Ebene bespielen, dann muss es konkrete Ideen der Makrosteuerung geben, und zwar solche, die entsprechend grundlegende Determinanten der sozioökonomischen Entwicklung beeinflussen (wie etwa das Steuersystem, ein mögliches Grundeinkommen, die neu diskutierte Bodenfrage, die Rolle von Wachstum als Treiber und Sachzwang ...). Und man muss eine Idee davon haben, wie sie umsetzbar sind und welche Wirkungen und Nebenwirkungen ihre Einführung hätte, insbesondere auch in sozialer Hinsicht (Blythe/Silver/Evans et al. 2018). Sollte sich der Eintritt in eine Postwachstumsära tatsächlich als "krisenhafte und mehrheitlich unerwünschte Folge des strukturellen Wandels« (Wiesenthal 2019: 379) herausstellen, sind vielschichtige Friktionen programmiert, auf die zu reagieren ist. Erst vor diesem Hintergrund ließe sich über konkrete Planungsaufgaben nachdenken, damit experimentieren und ließen sich diese ausprobieren. Dies bringt jedoch ein gewisses Dilem- 
ma für raumbezogene Diskurse mit sich, die aus guten Gründen eher kleinteilig denn großräumig gefasst sind. So lange Große Transformation aber die Makroebene auslässt (Thomasberger 2016) und stattdessen die Blaupause für Mikroprozesse liefern soll, dann wäre das höchstens Nachhaltigkeit in neuem Gewand. Dies würde Transformationen dienen, aber nicht notwendigerweise dem großen Umbau. Unter diesen Bedingungen würde sich der Mehrwert der großen Erzählung darin erschöpfen - um eine Formulierung von Tom Sieverts (2015: 19) zu nutzen -, Realität zu »verzaubern«. Sie würde diese Realität aber bei Weitem noch nicht fundamental verändern, und es ist auch unklar, ob sie in dieser Hinsicht besser geeignet wäre als bis dato diskutierte Konzepte wie etwa Nachhaltige Entwicklung. Insofern besteht die Gefahr, dass die Debatte um Transformation einen Hype erzeugt, der nicht von Dauer sein wird. Das Konzept der (Großen) Transformation muss sich im Dickicht der real ablaufenden Transformationen - der gewünschten, der nicht intendierten, der zufällig verlaufenden - meines Erachtens erst noch als zielführende Vision erweisen.

\section{Literatur}

Angelo, H.; Wachsmuth, D. (2015): Urbanizing urban political ecology: A critique of methodological cityism. In: International Journal of Urban and Regional Research 39 (1), 16-27.

Aring, J.; Sinz, M. (2006): Neue Leitbilder der Raumentwicklung in Deutschland: Modernisierung der Raumordnungspolitik im Diskurs. In: disP The Planning Review 42 (165), 43-60.

Beckenbach, F. (2017): Moderne Wirtschaftswissenschaften im Spannungsfeld von Paradigmaentwicklung und Problemlösungserwartungen. Keynote-Vortrag auf der IÖW-Tagung "Transformative Wirtschaftswissenschaft im Kontext Nachhaltiger Entwicklung«. Berlin, 06.11.2017.

Bettini, Y.; Arklay, T.; Head, B. W. (2017): Understanding the Policy Realities of Urban Transitions. In: Frantzeskaki, N.; Castán Brotoet, V.; Coenen, L.; Loorbach, D. (eds.): Urban Sustainability Transitions. London, 37-49. Blythe, J.; Silver, J.; Evans, L.; Armitage, D.; Bennett, N. J.; Moore, M. L.; Morrison, T. H.; Brown, K. (2018): The dark side of transformation: latent risks in contemporary sustainability discourse. In: Antipode 50 (5), 1206-1223. 
Brand, U. (2016): „Transformation« as a new critical orthodoxy: The strategic use of the term "Transformation « does not prevent multiple crises. In: GAIA-Ecological Perspectives for Science and Society 25 (1), 23-27.

Dahlstrom, M. F. (2014): Using narratives and storytelling to communicate science with nonexpert audiences. In: PNAS 111 (Supplement 4), 36143620.

Dörre, K.; Rosa, H.; Becker, K.; Bose, S.; Seyd, B. (eds.). (2019): Große Transformation? Zur Zukunft moderner Gesellschaften: Sonderband des Berliner Journals für Soziologie. Wiesbaden.

Frantzeskaki, N; Broto, V. C.; Coenen, L.; Loorbach, D. (eds.) (2017): Urban Sustainability Transitions. New York, Abingdon.

Giesel, K. D. (2007): Leitbilder in den Sozialwissenschaften. Begriffe, Theorien und Forschungskonzepte. Wiesbaden.

Grunwald, A. (2015): Transformative Wissenschaft - eine neue Ordnung im Wissenschaftsbetrieb? In: GAIA-Ecological Perspectives for Science and Society 24 (1), 17-20.

Grunwald, A. (2018): Transformative Wissenschaft als honest broker? Das passt! In: GAIA-Ecological Perspectives for Science and Society 27 (1), $113-116$.

Hesse, M.; Leick, A. (2013): Wachstum, Innovation, Metropolregionen. Zur Rekonstruktion des jüngeren Leitbildwandels in der deutschen Raumentwicklungspolitik. In: Raumforschung und Raumordnung 71 (4), 343359.

Iwaniec, D. M.; Cook, E. M.; Barbosa, O.; Grimm, N. B. (2019): The framing of urban sustainability transformations. In: Sustainability 11 (3), 573, 1-10.

Koch, F.; Kabisch, S.; Krellenberg, K. (2018): A Transformative Turn towards Sustainability in the Context of Urban-Related Studies? A Systematic Review from 1957 to 2016. In: Sustainability 10 (1), 58, 1-17.

Kollmorgen, R.; Merkel, W.; Wagener, H.-J. (2015): Handbuch Transformationsforschung. Wiesbaden.

Lamker, C.; Levin-Keitel, M. (2019): Planung im Wandel - von Rollenverständnissen und Selbstbildern. In: Raumforschung und Raumordnung 77 (2), 107-113.

Miessen, M. (2012): Albtraum Partizipation. Berlin.

Nassehi, A. (2019): »Ziele formulieren kann jeder. Wie kann man die Erderhitzung stoppen? Die einen glauben an Greta, die anderen an den Markt. Der Soziologe Armin Nassehi hat eine bessere Idee«. taz, 17.06.2019, 20-22. 
Pielke, R.A. (2007): The Honest Broker. Making Sense of Science in Policy and Politics. Cambridge.

Rodríguez-Pose, A. (2018): The revenge of the places that don't matter (and what to do about it). In: Cambridge Journal of Regions, Economy and Society 11 (1), 189-209.

Schneidewind, U. (2018): Die Große Transformation: Eine Einführung in die Kunst gesellschaftlichen Wandels. Frankfurt a. M.

Schön, D. A. (1983): The Reflective Practitioner: How Professionals Think in Action. New York.

Sieverts, T. (2015): Bedingungen der Stadtentwicklung heute: Das Beispiel Karlsruhe. In: Stadt Karlsruhe (Hg.): Auf dem Weg zum räumlichen Leitbild. Karlsruhe, 15-19.

Strunz, S.; Gawel, E. (2017): Transformative Wissenschaft: Eine kritische Bestandsaufnahme der Debatte. In: GAIA-Ecological Perspectives for Science and Society 26 (4), 321-325.

Thomasberger, C. (2016): Die Große Transformation und die Marktgesellschaft. In: Ökologisches Wirtschaften 31 (1), 30-34.

WBGU - Wissenschaftlicher Beirat Globale Umweltveränderungen (2011): Welt im Wandel. Gesellschaftsvertrag für eine große Transformation. Berlin.

WBGU - Wissenschaftlicher Beirat Globale Umweltveränderungen (2016): Der Umzug der Menschheit: Die transformative Kraft der Städte. Berlin. Wiesenthal, H. (2019): Institutionelle Transformationen gestern - und morgen? In: Dörre, K.; Rosa, H.; Becker, K.; Bose, S.; Seyd, B. (Hg.): Große Transformation? Zur Zukunft moderner Gesellschaften: Sonderband des Berliner Journals für Soziologie. Wiesbaden, 367-382.

Wissenschaftsrat (2015): Zum wissenschaftspolitischen Diskurs über Große gesellschaftliche Herausforderungen. Stattgart. = Positionspapier Drs. 4594-15.04.2015.

Zieschank, R.; Ronzheimer M. (2017): Große Transformation und die Medien - Alternativen zum Wachstum als Leitbild der Kommunikation. Dessau. $=$ UBA-Dokumentationen 12/2017.

Zolfagharian, M.; Walrave, B.; Raven, R.; Romme, A. G. L. (2019): Studying transitions: Past, present, and future. In: Research Policy 48 Issue 9. DOI: https://doi.org/10.1016/j.respol.2019.04.012 